\title{
The predictive value of cumulative toxicity for quality of life in patients with metastatic colorectal cancer during first-line palliative chemotherapy
}

This article was published in the following Dove Press journal: Cancer Management and Research

\author{
Claudia SEW \\ Schuurhuizen ${ }^{1,2}$ \\ Henk MW Verheul' \\ Annemarie MJ Braamse ${ }^{3}$ \\ Laurien M Buffart ${ }^{1-4}$ \\ Haiko J Bloemendal ${ }^{5}$ \\ Joost Dekker $2,6, *$ \\ Inge RHM Konings 1 ** \\ 'Department of Medical Oncology, \\ VU University Medical Center, Cancer \\ Center Amsterdam, Amsterdam, \\ The Netherlands; ' ${ }^{2}$ Department of \\ Psychiatry, Amsterdam Public Health \\ Research Institute, VU University \\ Medical Center, Amsterdam, The \\ Netherlands; ${ }^{3}$ Department of \\ Medical Psychology, Academic \\ Medical Center Amsterdam, Cancer \\ Center Amsterdam, Amsterdam, \\ The Netherlands; ${ }^{4}$ Department of \\ Epidemiology and Biostatistics, VU \\ University Medical Center, Cancer \\ Center Amsterdam, Amsterdam, \\ The Netherlands; ${ }^{5}$ Department of \\ Medical Oncology, Meander Medical \\ Center, Amersfoort, The Netherlands; \\ ${ }^{6}$ Department of Rehabilitation \\ Medicine, VU University Medical \\ Center, Cancer Center Amsterdam, \\ Amsterdam, The Netherlands \\ *These authors contributed equally to \\ this work
}

Correspondence: Henk MW Verheul Department of Medical Oncology, Cancer Center Amsterdam, VU University Medical Center, PO Box 7057, I08I HV, Amsterdam, The Netherlands

Tel +3I 20444432 I

Email h.verheul@vumc.nl
Background: Studies evaluating new systemic agents tend to report severe toxicities only, while the cumulative effect of multiple lower grade adverse events (AEs) may have an additional negative impact on patient quality of life (QOL). In the current observational cohort study, we evaluated whether, in patients with metastatic colorectal cancer receiving first-line chemotherapy, cumulative toxicity comprising all grades of AEs is more predictive for QOL than cumulative toxicity due to only high-grade AEs.

Methods: One hundred and five patients starting treatment completed the European Organization for the Research and Treatment of Cancer Quality of Life Questionnaire (EORTC-QLQC30) questionnaire at baseline and 10 weeks. AEs, clinical outcomes, and demographics were retrieved from patient records. Cumulative toxicity scores were calculated in three ways: total number of high-grade AEs, total number of all-grade AEs, and total number of AEs multiplied by their grade (the severity score). Relations between cumulative toxicity scores and QOL were studied using multivariable linear regression analyses.

Results: The mean age of patients was 65 years, $68 \%$ were male, and $84 \%$ received oxaliplatinbased chemotherapy. A higher total number of AEs of all grades $(\mathrm{B}=-2.4,95 \% \mathrm{CI}=-3.9 ;-0.9)$ and the severity score $(B=-1.4,95 \% \mathrm{CI}=-2.3 ;-0.5)$ were predictive for clinically relevant changes in physical QOL, whereas the total high-grade AEs was not. None of the cumulative toxicity scores were predictive for global QOL.

Conclusion: Cumulative toxicity scores comprising all grades of AEs provide a better measure of treatment burden than a toxicity score comprising high-grade AEs only. Physical QOL seems to be more affected by AEs than global QOL. Our results emphasize that future clinical trials should present cumulative toxicity scores comprising all AE grades as well as physical QOL instead of global QOL.

Keywords: cumulative toxicity, treatment-related toxicity, adverse events, quality of life, metastatic colorectal cancer

\section{Introduction}

Palliative systemic treatment regimens in patients with metastatic colorectal cancer (mCRC) are frequently accompanied by adverse events (AEs). Reporting AEs is a key component of oncological randomized controlled trials (RCTs) to evaluate patient safety, to improve clinicians' understanding of toxicity, and to assess risk-benefit ratios. ${ }^{1}$ AEs are graded by clinicians using the National Cancer Institute's Common Terminology Criteria for Adverse Events (NCI-CTCAE) on a five-point ordinal scale, with higher 
numbers being worse, and grades 3 and 4 generally indicating a need for clinical action. For instance, the following clinical descriptions match the different grades for vomiting: grade 1 , 1-2 episodes of vomiting in 24 hours; grade 2,3-5 episodes of vomiting in 24 hours; grade $3, \geq 6$ episodes of vomiting in 24 hours and indication for tube feeding, total parenteral nutrition or hospitalization, and grade 4, life-threatening consequences indicating urgent intervention. ${ }^{2}$ However, RCTs evaluating new systemic agents tend to limit reporting to severe toxicities only, presenting these as the pooled incidence of grades 3-4 AEs for the total study population. ${ }^{3}$ Consequently, lower grade toxic effects are often not taken into account. Nevertheless, these frequently long-lasting toxicities may have a major impact on a patient's quality of life (QOL). In a recent surveystudy, it was indeed demonstrated that a substantial number of patients were unwilling to undergo treatment because of anticipated grades 1 and 2 AEs. ${ }^{4}$ Furthermore, a recent cohort study showed that low-grade toxicity had a clinical impact on older patients receiving chemotherapy, and the accumulation of solely grades 1-2 AEs affecting these patients frequently led to treatment modification and discontinuation. ${ }^{5}$

Limited information is available on the burden represented by all (including low-grade) AEs experienced in RCTs. ${ }^{6-8}$ Several methods to improve toxicity reporting have been proposed. However, these approaches require access to specific software packages and an understanding of complex AE analyses, ${ }^{9,10}$ are retrospective in nature, ${ }^{9,10}$ are only applicable to specific (inpatient) cancer patients, ${ }^{6}$ or require an extensive monitoring system, ${ }^{6}$ all of which make them less practical for daily clinical use. Moreover, the majority of these existing approaches do not consider the effect of lower-grade AEs..$^{9,11}$

RCTs evaluating systemic treatments frequently use the two-item global QOL scale of the European Organization for the Research and Treatment of Cancer Quality of Life Questionnaire (EORTC QLQ-30) to assess QOL. ${ }^{12} \mathrm{We}$ previously reported that global QOL is unaffected by severe AEs due to palliative systemic treatment in patients with mCRC. ${ }^{13}$ Concern about the sensitivity of global QOL was also raised in a large cross-sectional study, which revealed that global QOL in patients with cancer was comparable with that of the general population. ${ }^{14}$ In contrast, functional and symptom scores were considerably worse in patients with cancer than in the general population. ${ }^{14}$ Therefore, global QOL may be less sensitive in detecting changes over time than functional QOL scales, ${ }^{15}$ and the impact of cumulative toxicity may be better reflected by physical QOL. ${ }^{13}$ Physical function or the ability to perform activities of daily living is an important aspect of QOL for patients with cancer. ${ }^{16}$
Furthermore, measures of physical QOL have been shown to be prognostic for survival. ${ }^{17}$

The purpose of this longitudinal cohort study was to evaluate the predictive impact of cumulative toxicity on physical and global QOL in patients with mCRC during the first 10 weeks of chemotherapy. We tested the following hypotheses: 1) cumulative toxicity comprising all grades of AEs (grades $1-4)$ is more predictive for QOL than cumulative toxicity involving only high-grade AEs (grades 3-4) and 2) cumulative toxicity is predictive for physical QOL, but does not (or less strongly) predict global QOL, in patients with $\mathrm{mCRC}$.

\section{Methods}

This is a secondary analysis on data obtained in the TES trial (Targeted screening, Enhanced, and Stepped care), a trial on the effectiveness of a combined screening and treatment program compared with usual care in reducing psychological distress in patients with mCRC. ${ }^{18}$ This study was approved by the Medical Ethics Committee of VU University Medical Center and registered in The Netherlands Trial Register (NTR4034). All patients provided written informed consent.

\section{Patients}

Patients were eligible if they were $\geq 18$ years old, diagnosed with $\mathrm{mCRC}$, and scheduled to receive first-line systemic treatment. They were recruited in two hospitals in The Netherlands between August 2013 and October 2016. Data on AEs were extracted from patient records, with complete data sets available for 105 patients.

\section{Source of primary data collection and measurements}

Clinicians reported AEs at every consultation, which occurred every 2-4 weeks after start of first-line systemic treatment, and additionally in case of emergency visits. Nonlaboratory AEs during the course of treatment were recorded by grade, as documented in patient records. When no grading of an AE was documented by the treating clinician, grading was assigned retrospectively using the NCI-CTCAE, version 4.0. This was done independently by two reviewers (CSEWS and AMJB), who were blinded to a patient's QOL rating. CTCAE items representing single AEs were graded on a five-point ordinal scale, with higher numbers being worse, and grades 3 and 4 generally indicating a need for clinical action. ${ }^{2}$ For each AE, the highest grade (from 1 to 5) was collected over the first 10 weeks of treatment.

Patients completed QOL questionnaires at baseline (prior to the start of first-line treatment) and after 10 weeks of 
treatment. Physical and global QOL were assessed with the EORTC QLQ-C30. This cancer-specific QOL questionnaire is internationally validated and widely used, ${ }^{12}$ including five items that represent physical functioning and two items that represent global QOL. Changes in QOL of at least 5-10 points are regarded as minimal clinically important differences. ${ }^{19,20}$ Additionally, patient demographics, tumor and treatment characteristics, and Eastern Cooperative Oncology Group Performance Score (ECOG PS) were identified from patient records. Clinical benefit during the first 10 weeks of chemotherapy treatment was evaluated by radiological response and was defined as partial response or stable disease upon CT evaluation.

\section{Statistical analysis}

Descriptive analyses were performed to summarize patient demographics, tumor, and treatment characteristics. Multivariable logistic regression analyses were used to compare characteristics of patients from the TES trial included in the current analyses with those patients who were excluded. To test the degree of agreement on grading AEs between the two reviewers, intraclass correlation coefficients (ICCs) were calculated using a two-way random effects model. Based on the $95 \%$ CI of the ICC estimate, values less than 0.5 , between 0.5 and 0.75 , between 0.75 and 0.9 , and greater than 0.9 are indicative of poor, moderate, good, and excellent reliability, respectively. ${ }^{21}$ The prevalence of specific types of AEs was calculated. Cumulative toxicity was assessed for each patient in three ways: 1) total number of severe AEs (grades 3-4); 2) total number of AEs (all grades); and 3) a severity score as the sum of the total number of AEs multiplied by their grade.

Associations between cumulative toxicity and physical and global QOL after 10 weeks of treatment were evaluated using linear regression analyses, adjusting for QOL at baseline. We built separate models for each of the three cumulative toxicity scores. In the multivariable regression models, we adjusted for age, gender (male vs female), clinical benefit after 10 weeks of treatment (yes vs no), type of chemotherapy (capecitabine vs CAPOX(-B) vs other regimen), number of chemotherapy cycles received, number of hospitalizations ( 0 vs 1 vs $\geq 2$ ), and allocation to treatment arm of the parent study (intervention vs control arm). Unstandardized regression coefficients (B) and 95\% CI were reported, indicating the change in QOL per unit cumulative toxicity, as well as standardized regression coefficients (beta) and $\mathrm{R}^{2}$ for each model. SPSS version 22 statistical software package was used for data analysis (IBM Corporation, Armonk, NY, USA).

\section{Results}

Patient characteristics are provided in Table 1. Among the 136 patients enrolled in two of the hospitals participating in the TES study, a total of 21 patients were not eligible for analyses in the present study due to missing QOL data after 10 weeks, leaving 105 eligible patients. Multivariable logistic regression analyses revealed no significant differences in baseline demographic and clinical characteristics between the 105 patients included and the 21 patients excluded from the analyses (data not shown). Mean (SD) age of included patients was $65 \pm 10$ years, $68 \%$ were male, and $84 \%$ received oxaliplatin-based chemotherapy. An ECOG PS was assigned to $80 \%$ of patients at start of systemic treatment, and of these, $76(95 \%)$ had ECOG PS $0-1$ and four (5\%) had ECOG PS 2 . For the majority of patients $(87 \%)$, palliative systemic treatment resulted in stable disease or a partial response, and $9 \%$ of patients had progressive disease at 10 weeks after start of treatment.

A total of 551 AEs were reported for all patients, 435 (78.9\%) of which had to be assigned retrospectively. The independent grading of AEs showed excellent reliability between the two reviewers (ICC $=0.971,95 \%$ CI 0.966; 0.976). In total, $103(98 \%)$ patients suffered from at least one $\mathrm{AE}$ (any grade), and 39 (37\%) patients experienced at least one high-grade AE. The remaining 64 (61\%) patients experienced exclusively low-grade AEs. The mean number of experienced AEs (all grades) was 5.3 \pm 2.7 . The mean number of high-grade AEs was $0.6 \pm 1.0$, and the mean severity score of AEs was $8.5 \pm 5.0$. The most common AEs (all grades) were neuropathy $(70 \%)$, diarrhea $(63 \%)$, and fatigue $(59 \%)$. Table 2 provides an overview of AEs that occurred in more than $10 \%$ of the study population. During the first 10 weeks after start of treatment, 38 patients $(36 \%)$ were admitted to hospital at least once. The most frequent reasons for hospitalization were diarrhea $(22 \%)$, fever (11\%), vomiting (10\%), and malaise (7\%).

\section{Cumulative toxicity and QOL}

A higher total number of all grades of $A E s(B=-2.4,95 \%$ $\mathrm{CI}=-3.9 ;-0.9)$ and a higher severity score $(\mathrm{B}=-1.4,95 \%$ $\mathrm{CI}=-2.3 ;-0.5)$ were both predictive for a significantly lower physical QOL (Table 3). The cumulative toxicity score measured by the total high-grade AEs was not predictive for a lower physical QOL. None of the cumulative toxicity scores were predictive for global QOL (Table 3).

\section{Discussion}

This longitudinal cohort study revealed that a cumulative toxicity score comprising all grades of AEs was more predictive for physical QOL in patients with $\mathrm{mCRC}$ receiving first-line 
Table I Patient characteristics

\begin{tabular}{|c|c|c|c|}
\hline$N=105$ n (\%) & & $\mathbf{N}(\%)$ & \\
\hline Gender & & Number of hospitalizations & \\
\hline male & 7I (67.6) & 0 & $67(63.8)$ \\
\hline \multirow[t]{2}{*}{ female } & $34(32.4)$ & I & $28(26.7)$ \\
\hline & & $\geq 2$ & $10(9.5)$ \\
\hline Age (years) & & Number of AEs & \\
\hline Mean (SD) & $64.8(9.7)$ & All grades nonlaboratory AEs (range) & $5.3(0-11)$ \\
\hline \multirow[t]{3}{*}{ Range } & $23-81$ & Laboratory AEs (range) & $5.1(0-13)$ \\
\hline & & Grades 3-4 nonlaboratory AEs (range) & $0.6(0-6)$ \\
\hline & & Laboratory AEs (range) & $0.3(0-3)$ \\
\hline Clinical benefit ${ }^{a}$ & & Severity score (range) & $8.5(0-25)$ \\
\hline No & $9(8.6)$ & & \\
\hline Yes & $91(86.7)$ & & \\
\hline Unknown & $5(4.8)$ & & \\
\hline Baseline ECOG PS & & Treatment modifications & \\
\hline 0 & $28(26.7)$ & Dose reduction & $62(59.0)$ \\
\hline 1 & $48(45.7)$ & Treatment delay & $45(42.9)$ \\
\hline 2 & $4(3.8)$ & Treatment switch & $7(6.7)$ \\
\hline Unknown & $25(23.8)$ & Treatment discontinuation & II (I0.5) \\
\hline Location of primary tumor & & Physical QOL score ${ }^{\mathrm{b}}$ & \\
\hline Right sided & $74(70.5)$ & Baseline (SD) & $74.9(21.0)$ \\
\hline \multirow[t]{2}{*}{ Left sided } & $31(29.5)$ & After 10 weeks (SD) & $72.3(22.6)$ \\
\hline & & Change in physical QOL (SD) & $2.54(19.81)$ \\
\hline Type of chemotherapy treatment & & Global QOL score & \\
\hline Capecitabine & $14(13.3)$ & Baseline (SD) & $62.7(24.1)$ \\
\hline CAPOX(-B) & $82(78.1)$ & After 10 weeks (SD) & $64.6(24.1)$ \\
\hline FOLFOX(-B) & $6(5.7)$ & Change in global QOL (SD) & $-1.90(21.75)$ \\
\hline Other & $3(2.9)$ & & \\
\hline
\end{tabular}

Notes: aClinical benefit was defined as partial response, stable disease vs progressive disease on CT evaluation. bScale ranges from 0 to 100.

Abbreviations: AEs, adverse events; ECOG PS, Eastern Cooperative Oncology Group Performance Score; QOL, quality of life.

Table 2 Incidence of the most common AEs ${ }^{a}$

\begin{tabular}{|c|c|c|c|c|c|}
\hline $\begin{array}{l}\text { AEs } \\
N=105\end{array}$ & $\begin{array}{l}\text { Grades } \\
1-2 N^{b}(\%)^{c}\end{array}$ & $\begin{array}{l}\text { Grades } \\
3-4 N^{b}(\%)^{c}\end{array}$ & Laboratory AEs & $\begin{array}{l}\text { Grades } \\
1-2 N^{b}(\%)^{c}\end{array}$ & $\begin{array}{l}\text { Grades } \\
3-4 N^{b}(\%)^{c}\end{array}$ \\
\hline$N=105$ & & & & & \\
\hline Gastrointestinal pain & $24(23)$ & $3(3)$ & Anemia & $77(73)$ & $I(I)$ \\
\hline Constipation & $12(11)$ & $\mathrm{I}(\mathrm{I})$ & Thrombocytopenia & $31(30)$ & - \\
\hline Pain, other & $17(16)$ & $\mathrm{I}(\mathrm{I})$ & White blood cells decreased & $28(27)$ & $\mathrm{I}(\mathrm{I})$ \\
\hline Diarrhea & $54(5 I)$ & $13(12)$ & Neutrophil count decreased & $16(15)$ & $4(4)$ \\
\hline Mucositis & $13(12)$ & $\mathrm{I}(\mathrm{I})$ & Hypocalcemia & $19(18)$ & - \\
\hline Nausea & $42(40)$ & $4(4)$ & Hyponatremia & $36(34)$ & $4(4)$ \\
\hline Vomiting & $29(28)$ & $5(5)$ & Hypokalemia & $15(14)$ & $2(2)$ \\
\hline Neuropathy (sensory) & $71(68)$ & $2(2)$ & ASAT increased & $53(50)$ & $I(I)$ \\
\hline Malaise & $38(36)$ & $2(2)$ & $A F$ increased & $38(36)$ & $\mathrm{I}(\mathrm{I})$ \\
\hline Anorexia & $34(32)$ & - & ALAT increased & $41(39)$ & $\mathrm{I}(\mathrm{I})$ \\
\hline Dyspnea & $12(11)$ & - & Bilirubin increased & $22(21)$ & - \\
\hline Fatigue & $60(57)$ & $2(2)$ & GGT increased & $42(39)$ & $9(9)$ \\
\hline Fever & $17(16)$ & $4(4)$ & GFR decreased & $22(21)$ & $4(4)$ \\
\hline \multirow[t]{2}{*}{ Hand Foot Syndrome } & $24(23)$ & $I(1)$ & Creatinine increased & $23(22)$ & $3(3)$ \\
\hline & & & Hypoalbuminemia & $46(44)$ & - \\
\hline
\end{tabular}

Notes: ${ }^{a} \mathrm{AEs}$ with an incidence of more than $10 \%$ in the study population. ${ }^{b}$ Number of patients experiencing the AE. cPercentage of patients experiencing the AE.

Abbreviations: AE, adverse event; AF, alkaline phosphatase; ALAT, alanine aminotransferase; ASAT, aspartate aminotransferase; GFR, glomerular filtration rate; GGT, gamma-glutamyl transferase.

chemotherapy than a cumulative toxicity score consisting of only grades 3-4 AEs. This applies to a cumulative toxicity score defined as the total of all $\mathrm{AE}$ grades, as well as to a score defined as the total number of AEs multiplied by their grade (severity score). In this group of patients, the presence of each distinct $\mathrm{AE}$ was associated with a 2.4 point lower physical 
Table 3 Cumulative toxicity scores and QOL outcomes after 10 weeks

\begin{tabular}{|c|c|c|c|c|c|c|c|c|}
\hline & \multicolumn{4}{|l|}{ Physical QOL } & \multicolumn{4}{|l|}{ Global QOL } \\
\hline & B (95\% Cl) & Beta & $\mathbf{R}^{2}$ & $P$-value & B (95\% Cl) & Beta & $\mathbf{R}^{2}$ & $P$-value \\
\hline \multicolumn{9}{|l|}{ Cumulative toxicity measures } \\
\hline \multicolumn{9}{|l|}{ adjusted for relevant covariates ${ }^{a}$} \\
\hline Sum of total number of grades $3-4$ AEs & $-3.040(-8.174 ; 2.095)$ & -0.132 & 0.454 & 0.243 & $-3.385(-9.034 ; 2.264)$ & -0.136 & 0.432 & 0.237 \\
\hline Sum of total number of all grades of AEs & $-2.414(-3.943 ;-0.885)$ & -0.284 & 0.501 & $0.002 *$ & $0.327(-1.476 ; 2.129)$ & 0.036 & 0.424 & 0.720 \\
\hline Severity score ${ }^{b}$ & $-1.373(-2.295 ;-0.452)$ & -0.300 & 0.495 & $0.004^{*}$ & $-0.207(-1.238 ; 0.869)$ & -0.042 & 0.424 & 0.703 \\
\hline \multicolumn{9}{|l|}{$\begin{array}{l}\text { Cumulative toxicity measures } \\
\text { unadjusted for covariates }\end{array}$} \\
\hline Sum of total number of grades $3-4$ AEs & $-1.004(-4.718 ; 2.709)$ & -0.043 & 0.337 & 0.593 & $-2.348(-6.252 ; 1.556)$ & -0.095 & 0.361 & 0.236 \\
\hline Sum of total number of all grades of $A E s$ & $-1.506(-2.839 ;-0.173)$ & -0.177 & 0.379 & $0.027^{*}$ & $0.410(-1.061 ; 1.880)$ & 0.045 & 0.354 & 0.582 \\
\hline Severity score ${ }^{b}$ & $-0.668(-1.390 ; 0.054)$ & -0.147 & 0.356 & 0.069 & $-0.155(-0.939 ; 0.629)$ & -0.032 & 0.353 & 0.695 \\
\hline
\end{tabular}

Notes: adjusted for the following covariates: age, gender, clinical benefit after 10 weeks of treatment (yes vs no), type of chemotherapy, number of chemotherapy cycles received, number of hospitalizations, and allocation to treatment arm of the parent study. 'bum of total number of all grades of AEs multiplied by their grade. *Statistical significance was concluded at the two-sided significance level of 0.05 for these results.

Abbreviations: AEs, adverse events; QOL, quality of life.

QOL, bearing in mind that these patients had an average of five AEs. This implies that an increase in cumulative toxicity defined as the total of all grades of AEs is predictive for a clinically relevant lower physical QOL (ie, exceeding five points). ${ }^{19,20}$ Similarly, the presence of each distinct grade of AE was associated with a 1.4 point lower physical QOL, against a backdrop of an average of almost nine all grades of AEs experienced by these patients. This indicates that cumulative toxicity, defined as the severity score, was also predictive for a clinically relevant lower physical QOL.

In addition to demonstrating the importance of low-grade AEs for patient QOL, these results question the accuracy of standard methods of toxicity reporting during systemic treatment, which still principally rely on high-grade AEs. The deficiency of the current approach is further emphasized by the finding that almost two thirds of patients exclusively experienced low-grade AEs.

Our outcomes suggest that improvement of treatmentrelated toxicity management through the reduction of the total number of AEs, with prominence given to low-grade AEs, may result in clinically relevant improvements in patients' physical QOL. Physicians should be made increasingly aware that addressing lower-grade AEs in RCTs is as important as higher-grade AEs for optimizing physical QOL.

As expected, no significant predictive association was found between cumulative toxicity and global QOL. These results confirm that global QOL may not be the ultimate measure of the impact of toxicity on a patient's QOL. ${ }^{22}$ Our results suggest that physical QOL outcomes may constitute a better measure of the patient toxicity burden. Indeed, shifting the focus to physical QOL - as opposed to global QOL - as a relevant marker of a therapy's effect has been suggested previously. ${ }^{16,23}$ Moreover, physical QOL was shown to be strongly related to toxicity outcomes in patients receiving radiotherapy. ${ }^{24}$

When interpreting the results of this study, it is important to consider the following points. First, we tested our hypotheses with a primary focus on treatment-related AEs, even though these are sometimes hard to distinguish from (pretreatment) symptoms of the disease. For instance, QOL may be disturbed by palliative chemotherapy due to treatment-induced toxicity, whereas disease-related symptoms may improve during treatment by stopping tumor growth. Hence, QOL measures may be influenced by cumulative AEs in opposite directions. Second, we collected clinicianreported toxicity data using the CTCAE classification. A lack of reporting standards for AEs in RCTs, and specifically for reporting grades 1 and 2 AEs, has been described, ${ }^{25}$ which may have resulted in underreporting of toxicity in the present study. Additionally, the proposed cumulative toxicity scores are based on multiple AE collection intervals summarized into a single AE profile by the use of the worst (highest) grade of each type of event that occurred in any risk interval, known as the worst-grade method. ${ }^{9}$ This method masks lower-grade AEs as well as multiple episodes of the highest grade of an event. ${ }^{9}$ Third, the absence of an effect of the high-grade cumulative toxicity score on QOL outcomes may have been partly caused by a lack of power, since analyses were conducted using a modest group size of 105 patients, of whom fewer than half reported grades 3-4 AEs. However, a $37 \%$ incidence of high-grade AEs is comparable with many Phase III RCTs conducted in patients with mCRC, ${ }^{26-28}$ supporting the representativeness of our sample. Fourth, the homogeneity of a study population included in a trial may affect the generalizability of the results. Cancer type and type 
of treatment may influence the findings related to cumulative toxicity and its predictive effect on QOL. The presence of more severe treatment-related toxicity is likely to strengthen the effect on QOL, especially in treatment regimens that are notoriously toxic, such as the STAMP-I cisplatin, cyclophosphamide, and carmustine combination treatment in breast cancer patients, which is associated with considerable morbidity and mortality. ${ }^{29}$ In patients receiving these types of regimens, it is likely that more cumulative toxicity will be present and therefore a greater deterioration in QOL.

To our knowledge, we are the first to develop a score that encapsulates the impact of cumulative toxicity during systemic treatment, and which is feasible in clinical practice. Previously developed approaches to reporting the total burden of AEs require a substantial infrastructure, ${ }^{6,9-11}$ which creates a significant barrier to implementation in routine clinical practice. In contrast, the data used to develop our cumulative toxicity scores can be gathered by any clinician or nurse at any (scheduled) visit and requires only a limited time investment. To complement the CTCAE and address some of the issues mentioned previously, a patient-reported outcome measurement system for toxicity has recently been developed.$^{30}$ Our cumulative toxicity scores could be further improved by incorporating toxicity as experienced by the patient and taking information regarding number of episodes, duration, and time of onset of individual AEs into account.

In conclusion, cumulative toxicity scores comprising all AE grades provide a better measure of treatment burden than a toxicity score based on high-grade AEs only. Physical QOL is more affected by AEs than global QOL. Our results highlight a need for future clinical trials to present cumulative toxicity scores that include all grades of $\mathrm{AE}$, in addition to describing physical QOL rather than global QOL.

\section{Disclosure}

JD and HV received a grant from the Alpe d'Huzes/Dutch Cancer Society (VU 2011-5279) during the conduction of the study. HV receives grants from Roche, Vitromics Healthcare System, Novartis, Immunovo B.V., Amgen, non-financial support from Pfizer, and honoraria from Boehringer Ingelheim. These resources were all received outside the submitted work. The authors report no other conflicts of interest in this work.

\section{References}

1. Seruga B, Templeton AJ, Badillo FE, et al. Under-reporting of harm in clinical trials. Lancet Oncol. 2016;17(5):e209-e219.

2. National Cancer Institute. Common Terminology Criteria for Adverse Events (CTCAE) Version 4.0. US Department of Health and Human Services; 2009. Available from: www.hrc.govt.nz/sites/default/files/ CTCAE\%20manual\%20-\%20DMCC.pdf. Accessed June 27, 2018.
3. Gilbert A, Ziegler L, Martland M, et al. Systematic review of radiation therapy toxicity reporting in randomized controlled trials of rectal cancer: a comparison of patient-reported outcomes and clinician toxicity reporting. Int J Radiat Oncol Biol Phys. 2015;92(3):555-567.

4. Castellanos EH, Chen SC, Drexler H, Horn L. Making the grade: the impact of low-grade toxicities on patient preference for treatment with novel agents. J Natl Compr Canc Netw. 2015;13(12):1490-1495.

5. Kalsi T, Babic-Illman G, Fields P, et al. The impact of low-grade toxicity in older people with cancer undergoing chemotherapy. Br J Cancer. 2014;111(12):2224-2228.

6. Koehler M, Fischer T, Kropf S, Frommer J. Quantitative tool to evaluate the somatic burden due to chemotherapy-induced adverse events: the somatic burden score. Support Care Cancer. 2014;22(11):3089-3099.

7. Péron J, Maillet D, Gan HK, Chen EX, You B. Adherence to CONSORT adverse event reporting guidelines in randomized clinical trials evaluating systemic cancer therapy: a systematic review. J Clin Oncol. 2013;31(31):3957-3963.

8. Pitrou I, Boutron I, Ahmad N, Ravaud P. Reporting of safety results in published reports of randomized controlled trials. Arch Intern Med. 2009;169(19):1756-1761.

9. Trotti A, Pajak TF, Gwede CK, et al. TAME: development of a new method for summarising adverse events of cancer treatment by the Radiation Therapy Oncology Group. Lancet Oncol. 2007;8(7):613-624.

10. Thanarajasingam G, Atherton PJ, Novotny PJ, et al. Longitudinal adverse event assessment in oncology clinical trials: the Toxicity over Time (ToxT) analysis of Alliance trials NCCTG N9741 and 979254. Lancet Oncol. 2016;17(5):663-670.

11. Cleeland CS, Zhao F, Chang VT, et al. The symptom burden of cancer: Evidence for a core set of cancer-related and treatment-related symptoms from the Eastern Cooperative Oncology Group Symptom Outcomes and Practice Patterns study. Cancer. 2013;119(24):4333-4340.

12. Aaronson NK, Ahmedzai S, Bergman B, et al. The European Organization for Research and Treatment of Cancer QLQ-C30: a quality-of-life instrument for use in international clinical trials in oncology. $\mathrm{J} \mathrm{Natl}$ Cancer Inst. 1993;85(5):365-376.

13. Schuurhuizen C, Braamse AMJ, Konings I, et al. Does severe toxicity affect global quality of life in patients with metastatic colorectal cancer during palliative systemic treatment? A systematic review. Ann Oncol. 2017;28(3):478-486.

14. Hinz A, Mehnert A, Dégi C, et al. The relationship between global and specific components of quality of life, assessed with the EORTC QLQC30 in a sample of 2019 cancer patients. Eur J Cancer Care (Engl). 2017;26(2):e12416.

15. Giesinger JM, Kieffer JM, Fayers PM, et al. Replication and validation of higher order models demonstrated that a summary score for the EORTC QLQ-C30 is robust. J Clin Epidemiol. 2016;69:79-88.

16. Kluetz PG, Slagle A, Papadopoulos EJ, et al. Focusing on core patientreported outcomes in cancer clinical trials: symptomatic adverse events, physical function, and disease-related symptoms. Clin Cancer Res. 2016;22(7):1553-1558.

17. Mol L, Ottevanger PB, Koopman M, Punt CJ. The prognostic value of WHO performance status in relation to quality of life in advanced colorectal cancer patients. Eur J Cancer. 2016;66:138-143.

18. Schuurhuizen CS, Braamse AM, Beekman AT, et al. Screening and treatment of psychological distress in patients with metastatic colorectal cancer: study protocol of the TES trial. BMC Cancer. 2015;15:302.

19. Ringash J, O’Sullivan B, Bezjak A, Redelmeier DA. Interpreting clinically significant changes in patient-reported outcomes. Cancer. 2007;110(1):196-202.

20. Osoba D, Rodrigues G, Myles J, Zee B, Pater J. Interpreting the significance of changes in health-related quality-of-life scores. J Clin Oncol. 1998;16(1):139-144.

21. Koo TK, Li MY. A Guideline of selecting and reporting intraclass correlation coefficients for reliability research. J Chiropr Med. 2016;15(2):155-163.

22. Hinz A, Einenkel J, Briest S, et al. Is it useful to calculate sum scores of the quality of life questionnaire EORTC QLQ-C30? Eur J Cancer Care. 2012;21(5):677-683. 
23. Friese CR, Harrison JM, Janz NK, et al. Treatment-associated toxicities reported by patients with early-stage invasive breast cancer. Cancer. 2017;123(11):1925-1934.

24. Jensen K, Bonde Jensen A, Grau C. The relationship between observerbased toxicity scoring and patient assessed symptom severity after treatment for head and neck cancer. A correlative cross sectional study of the DAHANCA toxicity scoring system and the EORTC quality of life questionnaires. Radiother Oncol. 2006;78(3):298-305.

25. Trotti A, Colevas AD, Setser A, Basch E. Patient-reported outcomes and the evolution of adverse event reporting in oncology. J Clin Oncol. 2007;25(32):5121-5127.

26. Tabernero J, Yoshino T, Cohn AL, et al. Ramucirumab versus placebo in combination with second-line FOLFIRI in patients with metastatic colorectal carcinoma that progressed during or after first-line therapy with bevacizumab, oxaliplatin, and a fluoropyrimidine (RAISE): a randomised, double-blind, multicentre, phase 3 study. Lancet Oncol. 2015;16(5):499-508.
27. Seymour MT, Thompson LC, Wasan HS, et al. Chemotherapy options in elderly and frail patients with metastatic colorectal cancer (MRC FOCUS2): an open-label, randomised factorial trial. Lancet. 2011;377(9779):1749-1759.

28. Price TJ, Peeters M, Kim TW, et al. Panitumumab versus cetuximab in patients with chemotherapy-refractory wild-type KRAS exon 2 metastatic colorectal cancer (ASPECCT): a randomised, multicentre, openlabel, non-inferiority phase 3 study. Lancet Oncol. 2014;15(6):569-579.

29. Rosti G, Ferrante P, Ledermann J, et al. High-dose chemotherapy for solid tumors: results of the EBMT. Crit Rev Oncol Hematol. 2002;41(2): 129-140.

30. Basch E, Reeve BB, Mitchell SA, et al. Development of the National Cancer Institute's patient-reported outcomes version of the common terminology criteria for adverse events (PRO-CTCAE). J Natl Cancer Inst. 2014;106(9):dju244.

\section{Cancer Management and Research}

\section{Publish your work in this journal}

Cancer Management and Research is an international, peer-reviewed open access journal focusing on cancer research and the optimal use of preventative and integrated treatment interventions to achieve improved outcomes, enhanced survival and quality of life for the cancer patient. The manuscript management system is completely online and includes

\section{Dovepress}

a very quick and fair peer-review system, which is all easy to use. Visit http://www.dovepress.com/testimonials.php to read real quotes from published authors. 